This item was submitted to Loughborough's Research Repository by the author.

Items in Figshare are protected by copyright, with all rights reserved, unless otherwise indicated.

\title{
Development and evaluation of new control algorithms for a mechanical golf swing device
}

\section{PLEASE CITE THE PUBLISHED VERSION}

http://dx.doi.org/10.1243/09596518JSCE425

\section{PUBLISHER}

Professional Engineering Publishing (@ IMechE)

VERSION

VoR (Version of Record)

\section{LICENCE}

CC BY-NC-ND 4.0

\section{REPOSITORY RECORD}

Harper, Thomas E., Jonathan R. Roberts, Roy Jones, and A.J. Carrott. 2019. "Development and Evaluation of New Control Algorithms for a Mechanical Golf Swing Device”. figshare. https://hdl.handle.net/2134/10856. 
This item was submitted to Loughborough's Institutional Repository (https://dspace.lboro.ac.uk/) by the author and is made available under the following Creative Commons Licence conditions.

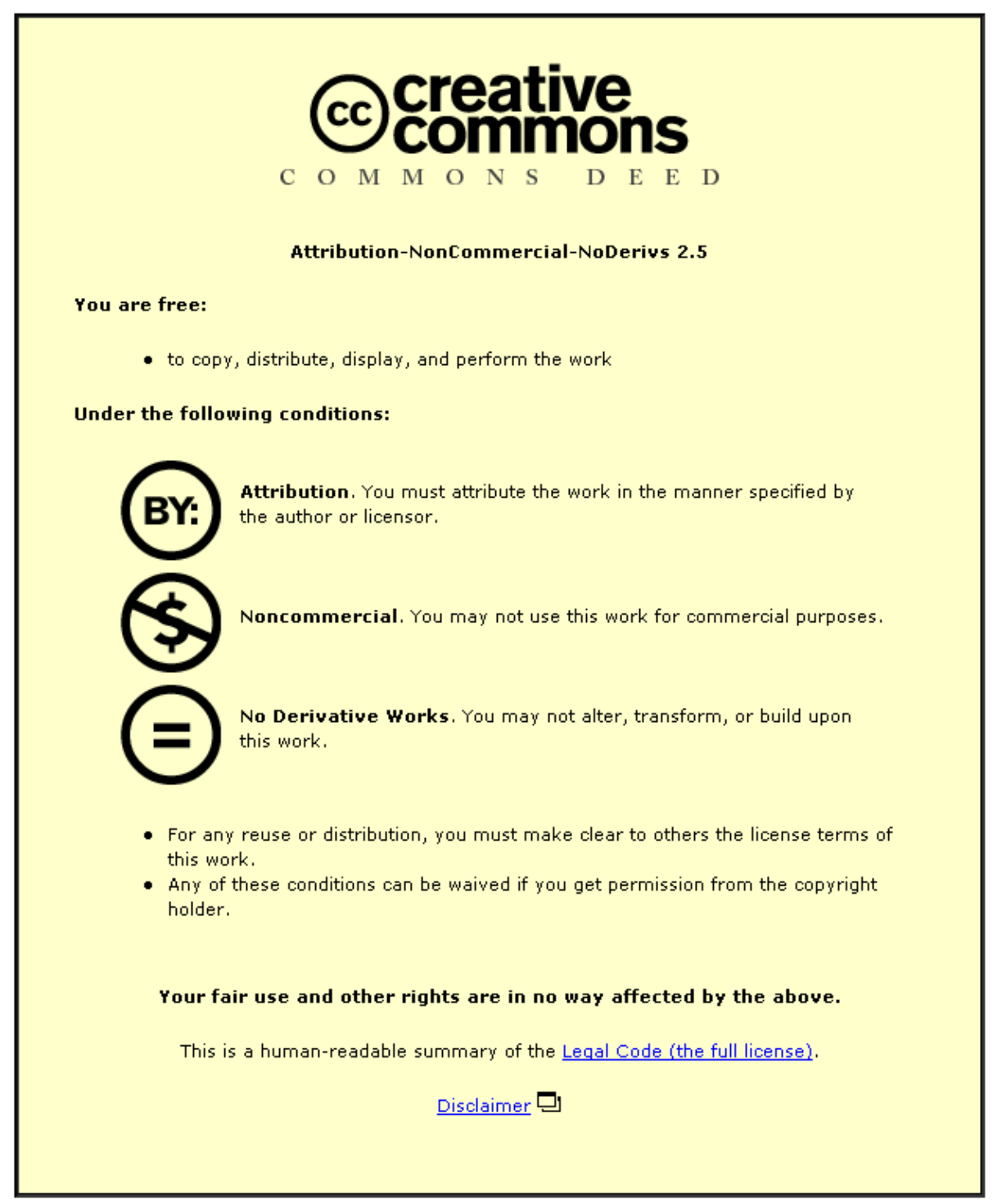

For the full text of this licence, please go to: http://creativecommons.org/licenses/by-nc-nd/2.5/ 


\title{
Development and evaluation of new control algorithms for a mechanical golf swing device
}

\author{
T Harper $^{1}$, J R Roberts ${ }^{1 *}$, R Jones ${ }^{1}$, and A J Carrott ${ }^{2}$ \\ ${ }^{1}$ Sports Technology Research Group, Loughborough University, Loughborough, UK \\ ${ }^{2}$ AJC Synectics Limited, Leicestershire, UK
}

The manuscript was received on 19 March 2007 and was accepted after revision for publication on 7 April 2008.

DOI: 10.1243/09596518JSCE425

\begin{abstract}
Golf swing machines have become fundamental tools in the development of new equipment because they provide more consistent swing motions than golfers. Golf robots perform a simplification of the complex sequence of motions that compose a golf swing; however, traditional devices are typically capable of performing only a single swing profile at variable speeds. Significant differences exist between individual golfers' swing motions, especially for golfers of different ability, experience, and physical stature, which suggests a requirement for swing profile variability in mechanical simulators. This investigation has found that the swing motion of a traditional golf robot provides a poor representation of golfers' swings and, as a result, a bespoke control system has been developed for a commercially available golf robot to enable performance of variable swing profiles with positional feedback. Robot swing command files are generated by fitting a curve to a number of discrete data points that are equally spaced in time, and which define angles representative of individual golfers' swings. The swing profiles of a professional golfer and a traditional golf robot were repeated accurately using this golf robot with a modified motion control system. The capability for individual golfers' swings to be accurately replicated using a mechanical device was demonstrated using feedback data. All manufacturers recognize the importance of tailoring equipment to the unique characteristics of a particular golfer's swing, and this increased robot functionality will provide considerable benefits in the development of customized equipment.
\end{abstract}

Keywords: golf robot, swing kinematics, motion control, swing simulation

\section{INTRODUCTION}

Golfers are used extensively by manufacturers for equipment development and testing purposes, where the players' opinions and perceptions are a valued source of information. There are, however, a number of limitations associated with player testing: golfers are inconsistent in performance, in opinion, and they tire. One method of overcoming these shortcomings lies in the development of golf swing simulation devices, which enable more consistent and controlled test conditions to be established for equipment research. Simple

*Corresponding author: Sports Technology Research Group, Wolfson School of Mechanical and Manufacturing Engineering, Loughborough University, Loughborough LE11 3TU, UK. email: J.R.Roberts@lboro.ac.uk mechanical devices date back to the 1920s, but the first 'advanced' golf robot was developed in 1966 by True Temper Sports, which performed a simplified swing motion based upon a double pendulum arrangement. Similar golf swing devices have been used increasingly over the last four decades to produce consistent, repeatable swings, and golf robots have become an intrinsic research instrument for manufacturers and governing bodies. The capability of smart structures to perform human dynamic motions is undoubtedly improving; however, the requirements for accurate simulations of complex motions, such as golf swings, still exceed current robotic capabilities. The objective of this study was to investigate the accuracy of swing motions performed by a traditional golf robot and to determine whether individual golfers' swings could be simulated accurately 
using a commercially available modern golf robot with a bespoke motion control system developed by Loughborough University and AJC Synectics.

\section{GOLF ROBOTS}

Golf robots perform a simplification of the complex sequence of motions that compose a golf swing. Simulated swing motions are typically based upon a planar double pendulum arrangement, which is inclined to represent golfers' swings, where the upper lever represents the golfers' arms and the lower lever represents the club [1]. The swing motion of the True Temper robot was based upon slow motion cinematic footage of professional golfer Byron Nelson's swing, and golf robots have since acquired the lasting moniker 'Iron Byron' [2]. The Miyamae Shot Robo III golf robot (Robo3) owned by Loughborough University's Sports Technology Research Group is typical of the majority of traditional golf robots. The Robo3's swing motion comprises three axes $-\theta_{\text {arm }}, \theta_{\text {wrist }}$, and $\theta_{\text {grip }}-$ that represent rotation of the arms, cocking of the wrists, and rotation of the club about the shaft axis respectively, as illustrated in Fig. 1. The angular position of the upper lever, $\theta_{\text {arm, }}$ is measured relative to the zero position or 'bottom dead centre' (BDC) when viewed orthogonally to the plane of motion. Similarly, $\theta_{\text {wrist }}$ is the angle formed between the upper lever and the lower lever, and $\theta_{\text {grip }}$ is the angle of rotation of the club about the shaft axis relative to a zero position at address. Clockwise rotations from BDC, which form the backswing, and the anticlockwise rotations from the top of the backswing until impact, which form the downswing, are assigned negative values, and the anticlockwise rotations, which form the followthrough, are assigned positive values.

The Robo3 is powered by a single $3 \mathrm{~kW}$ DC motor, which drives all three swing axes. The arm axis is powered directly using a belt drive system, and a gear mechanism provides motion to the lower lever at a ratio of 2:3 from $\theta_{\mathrm{arm}}$. A club-gripping mechanism is located at the distal end of the upper lever, which rigidly clamps the club during the swing. In addition, the gripping mechanism is geared to provide longitudinal rotation of the club about the shaft axis at a ratio of 3:5 from $\theta_{\text {arm }}$. A controlled range of clubhead speeds can be achieved by varying the motor drive speed; however, the geared nature of the Robo3's axes limits the robot to a single swing profile.

\section{SWING PROFILE MEASUREMENT AND ANALYSIS}

A 3D kinematic study of golfers' swings was conducted to determine the double pendulum joint rotations for each individual's swing. An automatic motion analysis system called Codamotion (CODA) was used to measure the motion of multiple marker locations to a high degree of accuracy $( \pm 0.05 \mathrm{~mm})$ at a capture frequency of $400 \mathrm{~Hz}$. Markers were strategically positioned on both the golfer and the club, which enabled a double pendulum model to be recreated in CODA for each golfer's swing, as illustrated in Fig. 2. Virtual markers were created, by means of fixed geometric relationships between two or more actual marker locations, at sites where actual markers would have been difficult to position. For example, a virtual marker was created at the midpoint between the shoulder markers to define the pivot $[3,4]$, while another was located at the midpoint of the golfer's left hand on the grip handle to define the wrist hinge [5]. A link between the virtual markers at the pivot and hinge was used to replicate the upper lever of the double pendulum model, and a link from the hinge to a marker on the shaft produced the lower lever. The angles $\theta_{\text {arm }}$ and $\theta_{\text {wrist }}$ were obtained by projecting the angles between the levers onto the $X Z^{\prime}$ plane (shown in Fig. 2(b)) which was inclined at an angle $\theta_{\text {swing }}$ to the horizontal. $\theta_{\text {swing }}$ was determined separately for each golfer using the angle of the shaft to the ground at impact as an approximation of that golfer's individual swing plane angle.

Angular data for $\theta_{\text {arm }}$ and $\theta_{\text {wrist }}$ were captured from five swings performed by each golfer and also by the Robo3. In this initial study only $\theta_{\text {arm }}$ and $\theta_{\text {wrist }}$ were considered because they contribute most to the main shape and power of the golf swing. These angular kinematic data, unique to individual golfers, were normalized by alignment of the measurement axis to the direction of the clubhead at impact and perpendicular to the shaft plane $\left(\theta_{\text {swing }}\right)$, and are referred to as a 'swing profile'.

One golfer was selected from each of the four following skill categories and their swing profiles were compared with the swing profile of the traditional golf robot: Professional, Category 1 (handicap <5); Category 2 (handicap 6-12); and Category 3 (handicap 13-20). Metrics calculated from the swing profile data along with clubhead speed at impact are summarized in Table 1. The metrics indicate that all golfers repeated their swings with high precision, achieving consistent $\theta_{\text {arm }}$ and $\theta_{\text {wrist }}$ values. The magnitude and timing of the 

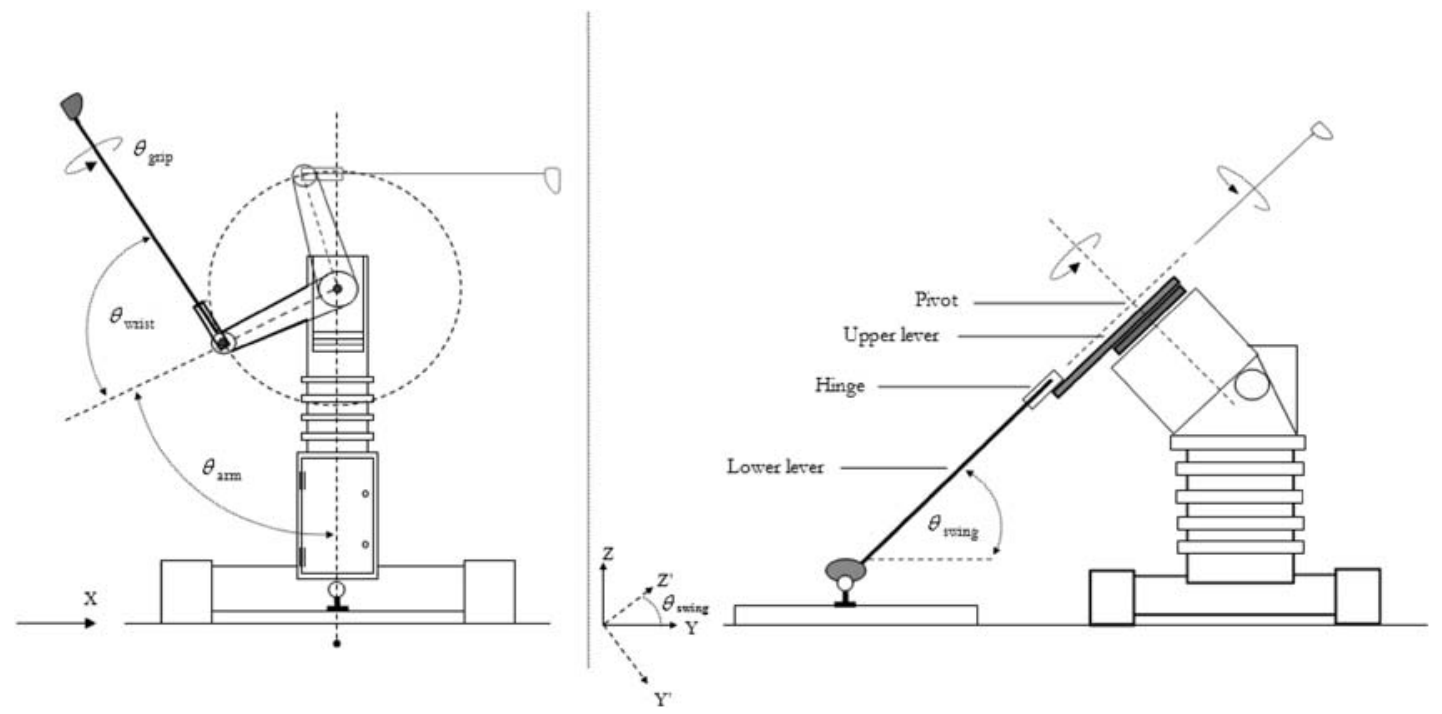

Fig. 1 Golf robot motion axes

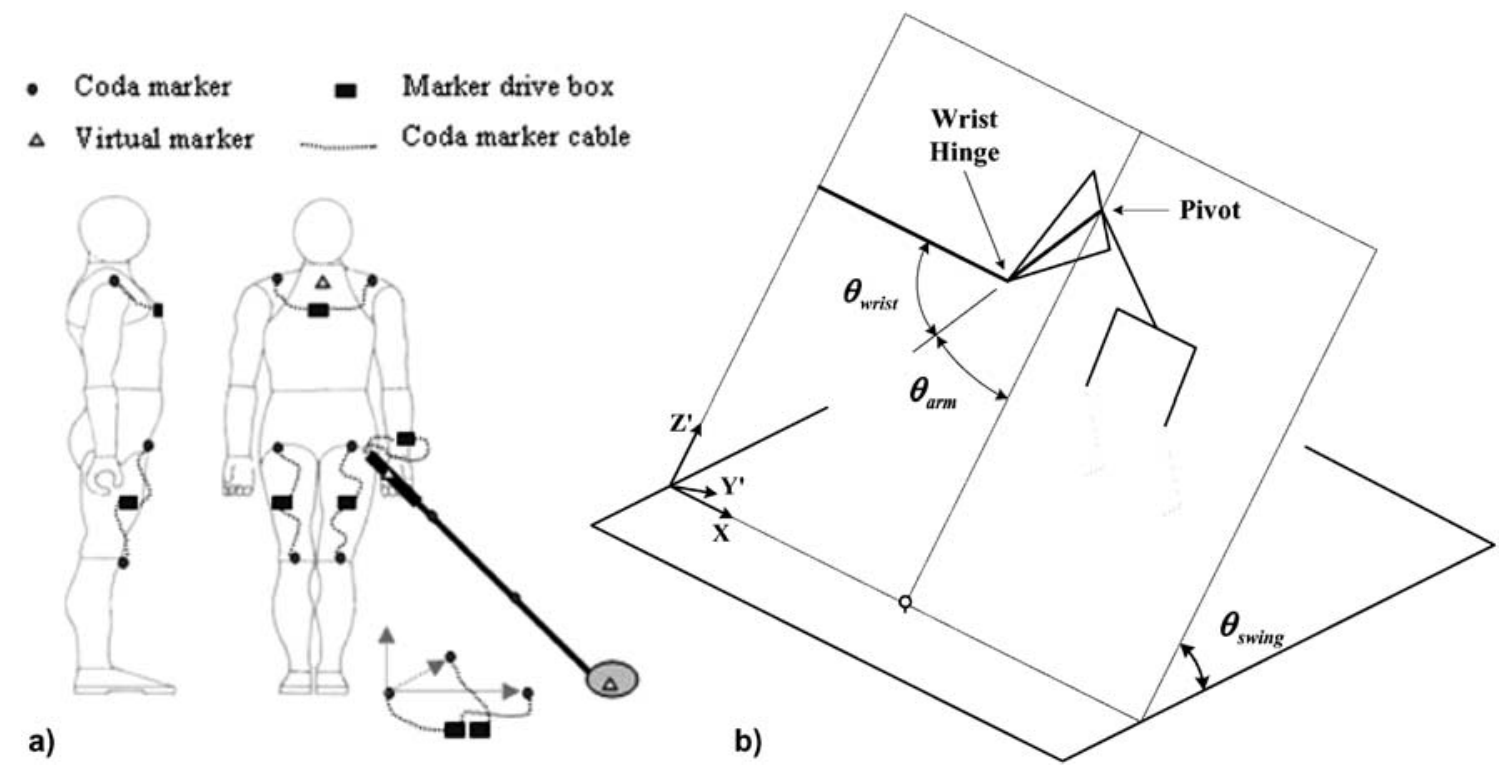

Fig. 2 (a) Marker locations used to recreate (b) the double pendulum model

measured swing angles, however, varied considerably between the individual golfers, which is attributable to the differences in the golfers' skill, age, experience, and physical stature.
A typical swing profile for each golfer was selected for further analysis, rather than calculating a profile mean, so that actual swings would be simulated later in the study. The swing profile of the Robo3 and the

Table 1 Mean and standard deviation of metrics calculated from the measured swing profiles

\begin{tabular}{|c|c|c|c|c|c|c|c|c|c|c|c|c|c|c|}
\hline \multirow[b]{3}{*}{ Swing type } & \multirow{2}{*}{\multicolumn{2}{|c|}{$\begin{array}{l}\text { Clubhead speed } \\
\text { at impact }\end{array}$}} & \multicolumn{4}{|c|}{ Peak swing profile angle } & \multicolumn{8}{|c|}{ Swing duration } \\
\hline & & & \multicolumn{2}{|c|}{$\theta_{\text {arm }}$} & \multicolumn{2}{|c|}{$\theta_{\text {wrist }}$} & \multicolumn{2}{|c|}{ Swing } & \multicolumn{2}{|c|}{ Backswing } & \multicolumn{2}{|c|}{ Downswing } & \multicolumn{2}{|c|}{ Wrist delay } \\
\hline & $(\mathrm{m} / \mathrm{s})$ & st dev & (deg) & st dev & (deg) & st dev & (s) & st dev & $(\mathrm{s})$ & st dev & (s) & st dev & (s) & st dev \\
\hline Robot & 50.7 & 0.1 & -148.3 & 0.1 & -89.3 & 0.0 & 4.305 & 0.01 & 3.815 & 0.00 & 0.378 & 0.00 & 0.000 & 0.00 \\
\hline Professional & 45.9 & 0.7 & -152.2 & 1.5 & -121.9 & 1.3 & 1.075 & 0.01 & 0.729 & 0.01 & 0.288 & 0.01 & 0.169 & 0.01 \\
\hline Cat 1 & 47.9 & 0.9 & -146.4 & 1.9 & -140.0 & 1.8 & 0.826 & 0.01 & 0.522 & 0.02 & 0.257 & 0.01 & 0.148 & 0.02 \\
\hline Cat 2 & 40.7 & 0.8 & -129.0 & 3.6 & -136.1 & 2.2 & 1.080 & 0.02 & 0.720 & 0.03 & 0.294 & 0.01 & 0.023 & 0.01 \\
\hline Cat 3 & 41.3 & 0.9 & -117.3 & 4.0 & -150.8 & 2.3 & 1.023 & 0.02 & 0.618 & 0.02 & 0.347 & 0.02 & 0.073 & 0.02 \\
\hline
\end{tabular}




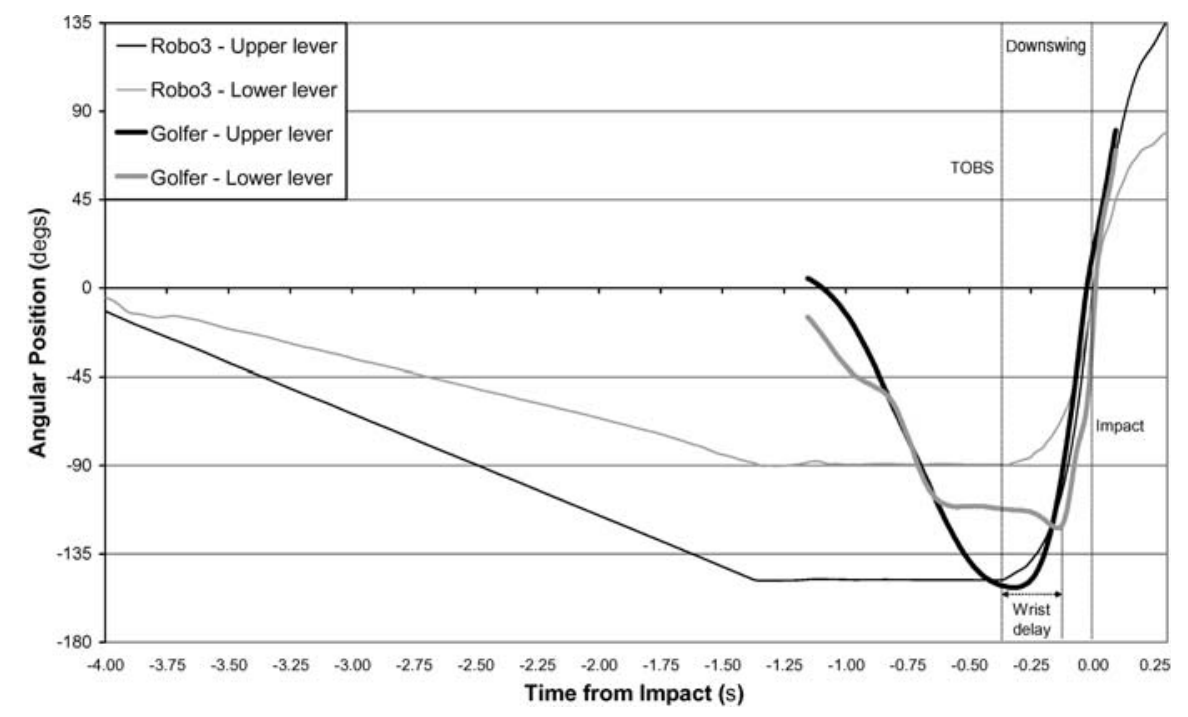

Fig. 3 Comparison of the kinematic data measured from the Robo3 and the professional golfer

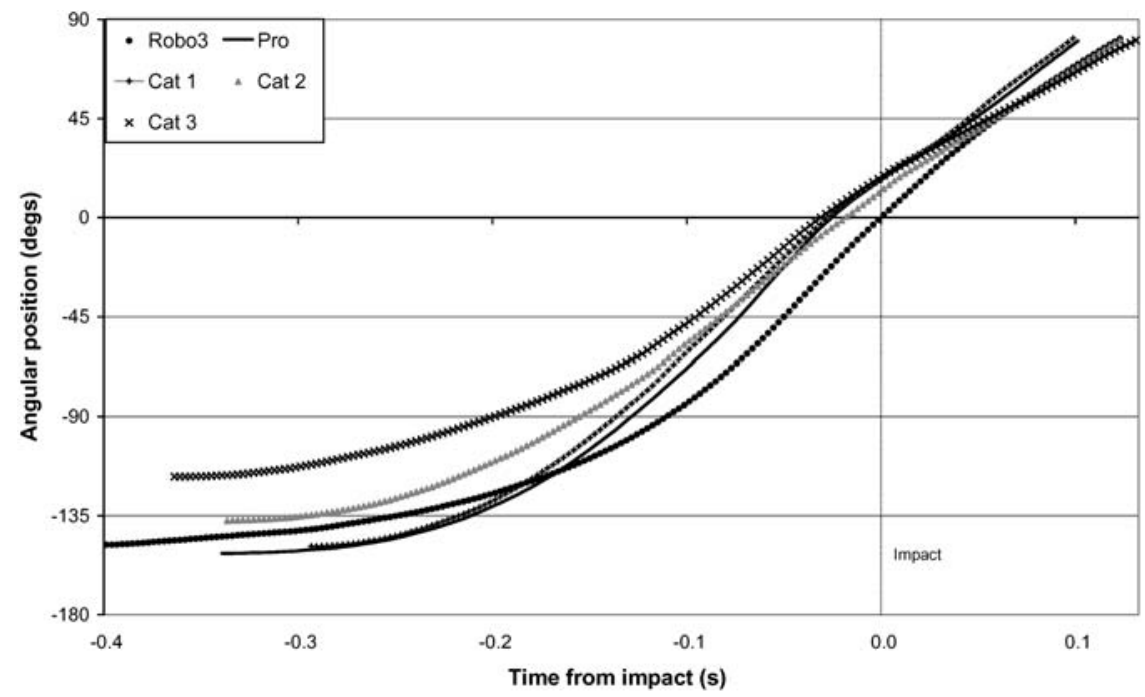

Fig. 4 Comparison of $\theta_{\text {arm }}$ values measured during the downswing

professional golfer are shown in Fig. 3; the profile traces have been synchronized to impact. A large difference in the profile duration was found to exist between the Robo3 and the golfers, as shown in Table 1 and Fig. 3; however, the downswing phases of the swings exhibited similar ranges of motion and duration. Figures 4 and 5 compare the $\theta_{\text {arm }}$ and $\theta_{\text {wrist }}$ profiles for the downswing of the Robo3's and the golfers' swings; these profiles were again synchronized to impact.

Figure 4 shows that the Robo3 provides a reasonable representation of the golfers' $\theta_{\text {arm }}$ motion during the downswing; however, angular differences of up to $30^{\circ}$ indicate that a single robot swing profile can not accurately represent the arm motion of all golfers. Figure 5 shows that the $\theta_{\text {wrist }}$ motion performed by the Robo3 is less representative of the golfers' wrist motion. The wrist axis of the Robo3 is geared, forcing $\theta_{\text {wrist }}$ to be $2 / 3$ of $\theta_{\text {arm }}$, and thus limiting the Robo3 to a maximum wrist-cock angle of $-90^{\circ}$. Golfers, however, were found to achieve peak $\theta_{\text {wrist }}$ angles from $-120^{\circ}$ to $-150^{\circ}$ at the top of the backswing (TOBS), which is significantly greater than the previously reported $-90^{\circ}$ approximation [1]. A delay between the commencement of downward motions in $\theta_{\text {arm }}$ and $\theta_{\text {wrist }}$ is also evident in the golfers' swing profiles, which is not present in the Robo3 profile owing to the gearing. Golfing literature refers to this offset as a 'delayed wrist release' $[\mathbf{1}, \mathbf{6}]$. Many sports motions, including the golf swing, employ a proximal to distal sequencing of joint segments to achieve maximal velocity of the 


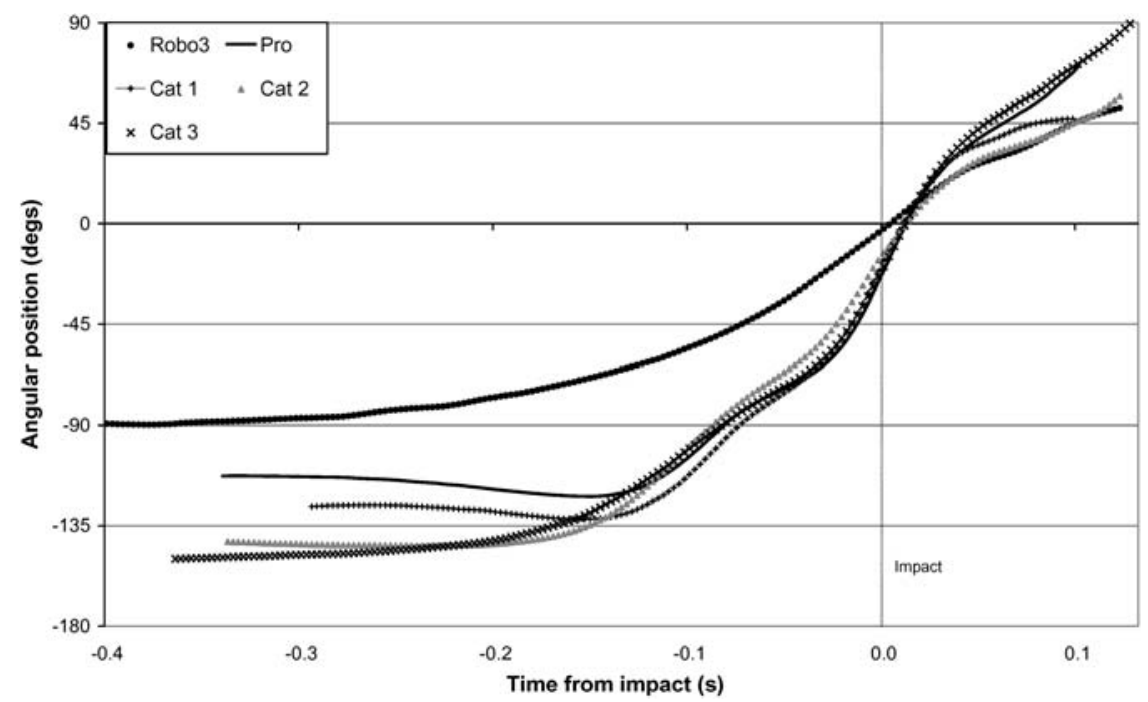

Fig. 5 Comparison of $\theta_{\text {wrist }}$ values measured during the downswing

distal end of the linked system, called the summation of speed principle $[\mathbf{7}, \mathbf{8}]$. Thus, for a golf robot to perform swing motions that represent golfers' swings more accurately, the device must be both capable of independent axes control to represent individual swing motions and able to perform critical swing characteristics such as the delayed wrist release.

\section{MODERN GOLF ROBOTS}

The Miyamae Shot Robo V golf robot (Robo5) is an advanced golf robot that has superseded the Robo3. The basic structure of the Robo5 is similar to the Robo3, but the Robo5 - marketed as 'the world's first controllable swing robot' - has independently driven motion axes, which enable variable swing profiles to be performed representative of different golfers and shot types. As a result, the Robo5 offers much greater performance potential than traditional golf simulators, but the device's graphical motion planning interface is difficult to program accurately and the lack of feedback data supplied by the Robo5's motion control system means that these inaccuracies will be difficult to detect and may go unnoticed.

\subsection{System modification}

The motion control system of the Robo5 was modified by AJC Synectics to provide increased levels of swing motion programmability. Encoders were fitted to the existing servomotors to provide positional feedback at a sampling rate of $250 \mathrm{~Hz}$, which could be downloaded and compared against the input swing command data. Bespoke motion planning software called Profile Designer (PD) was developed for the Robo5's new motion control system, which facilitated the generation and management of swing commands. The principle behind $\mathrm{PD}$ is to produce command files comprising one thousand angular joint positions for each motion axis by interpolating between a much smaller number of equally spaced discrete data points that describe the basic shape of the swing profile; a number of curve fitting algorithms can be used to perform this task. PD also provides a basic level of motion characterization for swing command files, where the position, velocity, and acceleration of motion axes are presented as a function of time in a graphical format, with derivative values calculated from the curves fitted to the positional data.

\section{EVALUATION OF THE NEW MOTION CONTROL SYSTEM}

Swing profile data from the Robo3 and the professional golfer were selected for replication using the modified Robo5. Five robot swing commands were generated for each swing profile using the different curve fit options available within PD: linear, Bezier, cubic spline to position, cubic spline to velocity, and cubic spline to acceleration. The backswing of the Robo3 swing profile was manually edited to remove the pause between the TOBS and commencement of the downswing. 


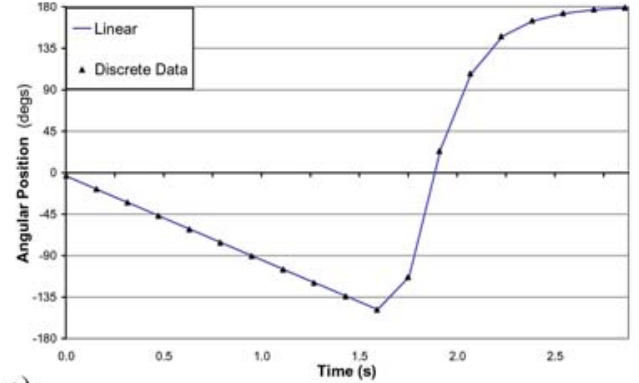

a)

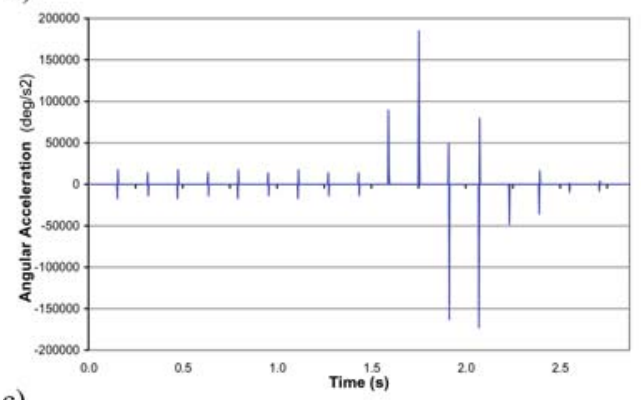

c)

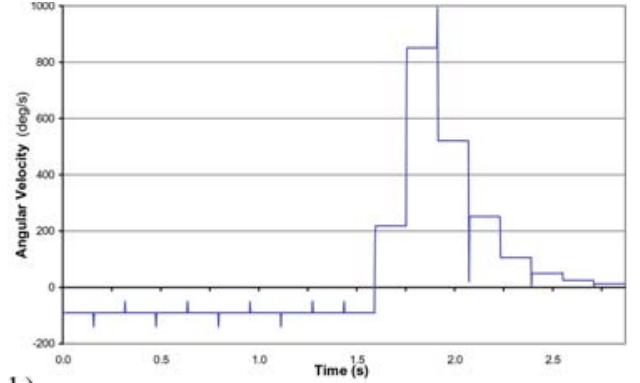

b)

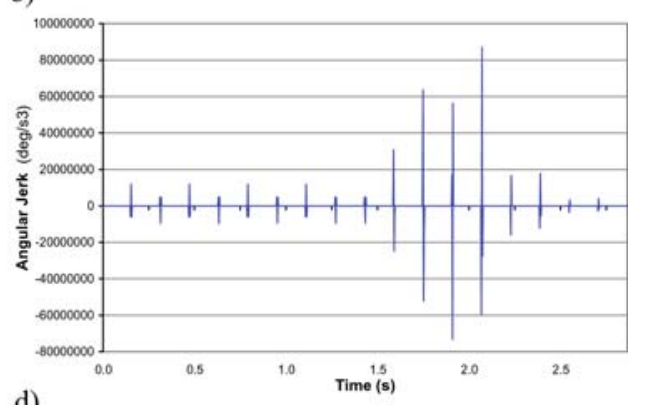

d)

Fig. 6 (a) Position, (b) velocity, (c) acceleration, (d) jerk of a Robo5 command file calculated by PD using a linear fit to discrete $\theta_{\text {arm }}$ data taken from the Robo3 swing profile

\subsection{Swing command generation}

Two characteristics determine the quality of the curves generated using PD: curve smoothness and closeness of fit. Curve smoothness is defined as the quality of being free from errors or interruptions, and a smooth function is therefore considered to be infinitely differentiable [9]. The quality of simulated motions performed by mechanical devices is governed by the smoothness of the first three derivatives of the command positions [10]. Sudden changes of gradient in the command position profile will require massive accelerations to produce these instantaneous changes in velocity. Therefore, it is desirable for Robo5 command files to contain smooth profile derivatives, and thus, minimize the likelihood of convulsive simulation motion. The closeness of fit describes the proximity of the fitted curve to the discrete joint positions and consequently it influences the accuracy of the simulated motion.

The linear algorithm connects adjacent joint positions with a straight line; thus, all of the discrete coordinates are satisfied by the interpolant curve, as illustrated in Fig. 6. The smoothness of the linear curve is poor, because large changes in gradient occur at the discrete joint positions. This results in instantaneous changes in the velocity profile and therefore massive impulsive accelerations, as illustrated in Figs 6(b) and 6(c). Instantaneous spikes in the derivative profiles are likely to result in convulsive Robo5 motions, and the quality of the swing simulations performed by the Robo5 was found to be poor using the linear algorithm.

The Bezier method for creating curves uses four control points to influence the shape of the fitted curve. The first and last points in the Bezier control sequence are satisfied by the interpolant curve (anchors), while the intermediate points (handles) act as attractors to influence the curve path between the anchors. The Bezier construction method enables easy and predictable manipulation of curve paths and has been used extensively in computer graphics software. PD sequentially considers the discrete joint positions as anchor and handle locations, e.g. 1-4 then 2-5, 3-6, and so on, but because the Bezier algorithm only satisfies anchor locations the closeness of fit of the interpolant curve is poor. The Bezier algorithm offers improved smoothness over the linear fit; however, instantaneous changes in profile gradient are still present in higher-order derivative profiles, which are again likely to result in undesirable robot motions.

The final curve fitting algorithm offered by PD is a third-order polynomial function called a cubic spline, which provides a smooth continuous curve for the object and first derivative data, as illustrated in Fig. 7. The second derivative has a linear profile shape and the third derivative a stepped profile shape. However, the magnitude of the third derivative peaks is considerably less than that generated using the linear and Bezier algorithms. Cubic splines are popular choices for smoothing noisy data or interpolating 

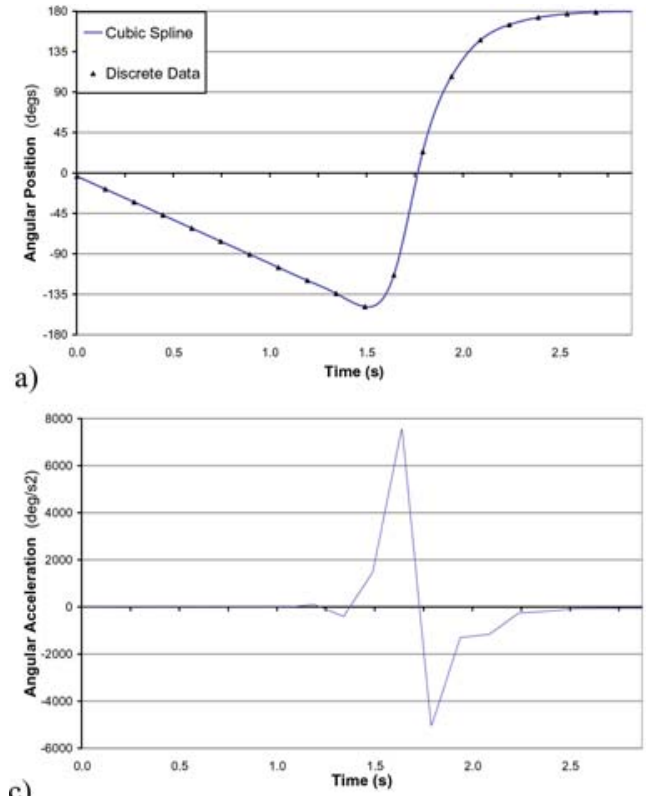

c)

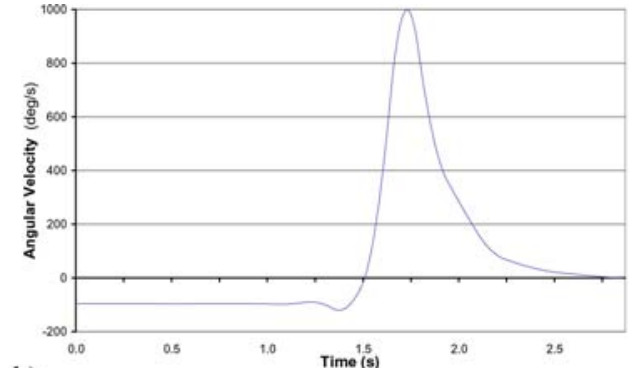

b)

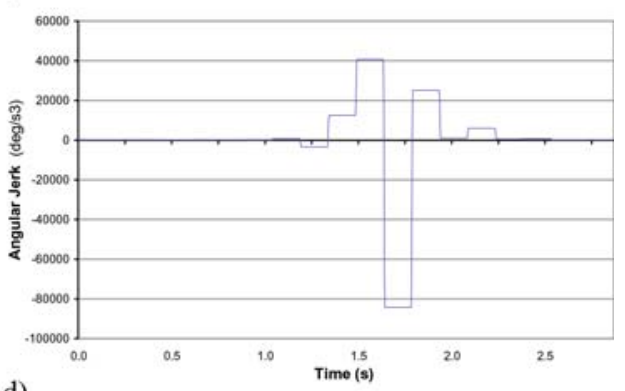

d)

Fig. 7 (a) Position, (b) velocity, (c) acceleration, (d) jerk of a Robo5 command file calculated by PD using a cubic spline fit to discrete $\theta_{\text {arm }}$ data taken from the Robo3 swing profile
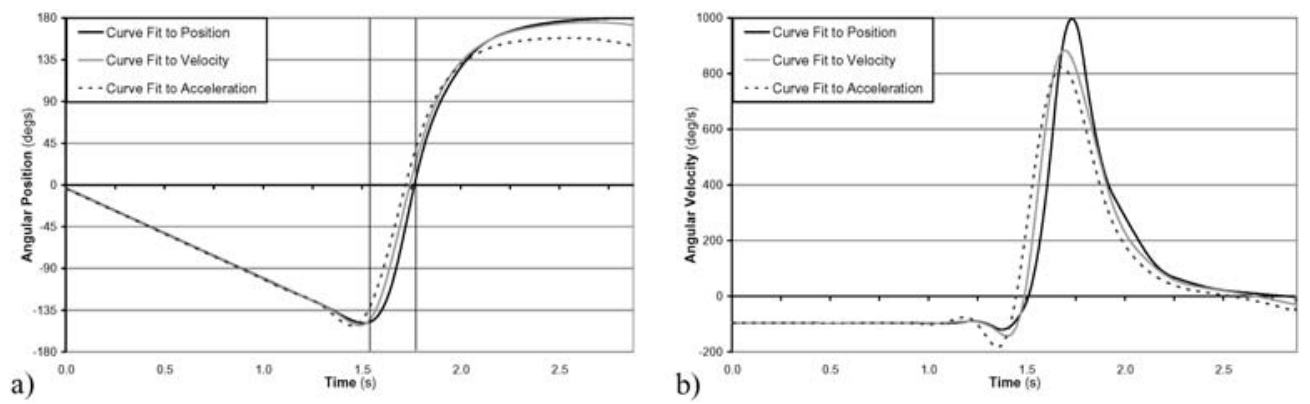

Fig. 8 Comparison of (a) angular position and (b) angular velocity profiles resulting from cubic spline curve fits to position, velocity, and acceleration data

between data points because they offer a good balance between simplicity and smoothness [11]. In PD, the spline fit may also be applied to either of the first two derivatives to increase the smoothness of the higher derivative profiles. The positional coordinates are then calculated by integration of the derivative profiles. As a result, the closeness of fit of the positional data to the discrete joint positions tends to be reduced, as illustrated in Fig. 8(a), while the peak velocity typically decreases as shown in Fig. 8(b). (In Fig. 8, the curve fit to position satisfies all the discrete data points as shown in Fig. 7(a)). The magnitude of the positional error and the velocity decrease arising from derivative fits has been found to be dependent upon the number of discrete data points, the profile shape, and the order of the derivative data to which the curve fit is applied.

\subsection{Swing simulations}

Three Robo5 command files were generated from the Robo3 and the professional golfer's swing profile data using the cubic spline algorithm applied to position, velocity, and acceleration in PD. Clubhead speeds at impact of $40 \mathrm{~m} / \mathrm{s}, 45 \mathrm{~m} / \mathrm{s}$, and $50 \mathrm{~m} / \mathrm{s}$ were selected to be representative of golfers' clubhead speeds. The swing durations that provided these clubhead speeds for the command files generated by a position fit were determined by trial and error. The clubhead speed generated by a Robo5 swing simulation is controlled by adjusting the time period that a swing command file is completed within. These same durations were also used for the command files obtained from the velocity and acceleration fits. The golf ball teeing position was manipulated for each swing simulation 


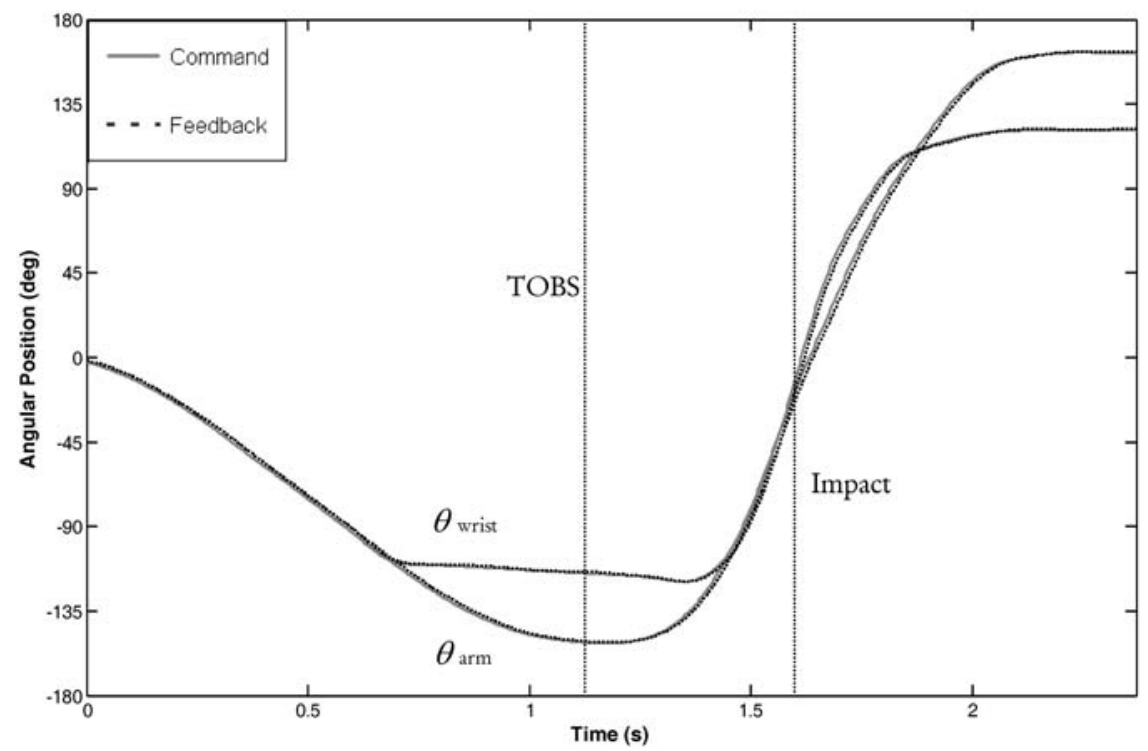

Fig. 9 Angular positions achieved by the Robo5 (feedback data) compared with the command file for the simulation of the professional golfer's swing

to ensure identical impact locations at the approximate geometric centre of the clubface. Three swing simulations were performed for each swing command file at each clubhead speed, and feedback data were downloaded from the Robo5's motion controller after each swing. The simulation results are summarized in Table 2 while Figs 9, 10, and 11 illustrate the simulation accuracy of the professional golfer's swing at an impact speed of $45 \mathrm{~m} / \mathrm{s}$. Figures 9 and 10 compare the swing command data and the motion feedback data in terms of angular position and velocity respectively. In addition, the angular differences between the command data and the feedback data (residual data) are plotted in Fig. 11, where positive values indicate that the swing positions performed by the Robo5 lag behind the swing command positions during clockwise rotations and are ahead during the anticlockwise rotations.

\section{DISCUSSION}

The command and feedback joint position data shown in Fig. 9 demonstrate that the Robo5 achieved a high level of positional accuracy, and this was consistent for all swing simulations. The

Table 2 Robo5 simulation data

\begin{tabular}{|c|c|c|c|c|c|c|c|c|c|}
\hline \multirow[b]{3}{*}{ Golfer } & \multirow[b]{3}{*}{ Cubic spline fit } & \multirow{3}{*}{$\begin{array}{l}\text { Target } \\
\text { clubhead } \\
\text { speed } \\
(\mathrm{m} / \mathrm{s})\end{array}$} & \multirow{3}{*}{$\begin{array}{l}\text { Profile } \\
\text { duration }\end{array}$} & \multirow{2}{*}{\multicolumn{2}{|c|}{$\begin{array}{l}\text { Actual clubhead } \\
\text { speed }\end{array}$}} & \multicolumn{4}{|c|}{ Mean absolute deviation } \\
\hline & & & & & & \multicolumn{2}{|c|}{$\theta_{\mathrm{arm}}$} & \multicolumn{2}{|c|}{$\theta_{\text {wrist }}$} \\
\hline & & & & $(\mathrm{m} / \mathrm{s})$ & st dev & (deg) & st dev & (deg) & st dev \\
\hline \multirow[t]{3}{*}{ Professional golfer } & Position & 40 & 2.370 & 40.0 & 0.0 & 1.5 & 0.0001 & 1.1 & 0.0001 \\
\hline & & 45 & 2.030 & 45.0 & 0.1 & 1.7 & 0.0002 & 1.2 & 0.0001 \\
\hline & & 50 & 1.900 & 50.1 & 0.1 & 0.2 & 0.0002 & 1.1 & 0.0004 \\
\hline \multirow[t]{3}{*}{ Professional golfer } & Velocity & 40 & 2.370 & 39.1 & 0.1 & 1.6 & 0.0974 & 1.3 & 0.0628 \\
\hline & & 45 & 2.030 & 44.0 & 0.0 & 1.8 & 0.0001 & 1.4 & 0.0000 \\
\hline & & 50 & 1.900 & 49.7 & 0.0 & 1.6 & 0.0000 & 1.3 & 0.0001 \\
\hline \multirow[t]{3}{*}{ Professional golfer } & Acceleration & 40 & 2.370 & 38.4 & 0.1 & 1.6 & 0.0001 & 1.3 & 0.0001 \\
\hline & & 45 & 2.030 & 44.3 & 0.1 & 1.7 & 0.0002 & 1.4 & 0.0001 \\
\hline & & 50 & 1.900 & 48.5 & 0.1 & 1.6 & 0.0001 & 1.2 & 0.0002 \\
\hline \multirow[t]{3}{*}{ Robo3 } & Position & 40 & 3.475 & 40.0 & 0.0 & 0.9 & 0.0000 & 0.6 & 0.0003 \\
\hline & & 45 & 3.165 & 45.0 & 0.0 & 1.1 & 0.0000 & 0.7 & 0.0000 \\
\hline & & 50 & 2.895 & 49.9 & 0.0 & 1.0 & 0.0001 & 0.6 & 0.0002 \\
\hline \multirow[t]{2}{*}{ Robo3 } & Velocity & 40 & 3.475 & 28.4 & 0.0 & 1.2 & 0.0000 & 0.7 & 0.0004 \\
\hline & & 50 & 2.895 & 36.8 & 0.0 & 1.3 & 0.0000 & 0.8 & 0.0001 \\
\hline \multirow[t]{3}{*}{ Robo3 } & Acceleration & 40 & 3.475 & 28.1 & 0.1 & 1.2 & 0.0001 & 0.7 & 0.0002 \\
\hline & & 45 & 3.165 & 29.5 & 0.0 & 1.4 & 0.0001 & 0.8 & 0.0002 \\
\hline & & 50 & 2.895 & 27.8 & 0.0 & 1.4 & 0.0001 & 0.9 & 0.0004 \\
\hline
\end{tabular}




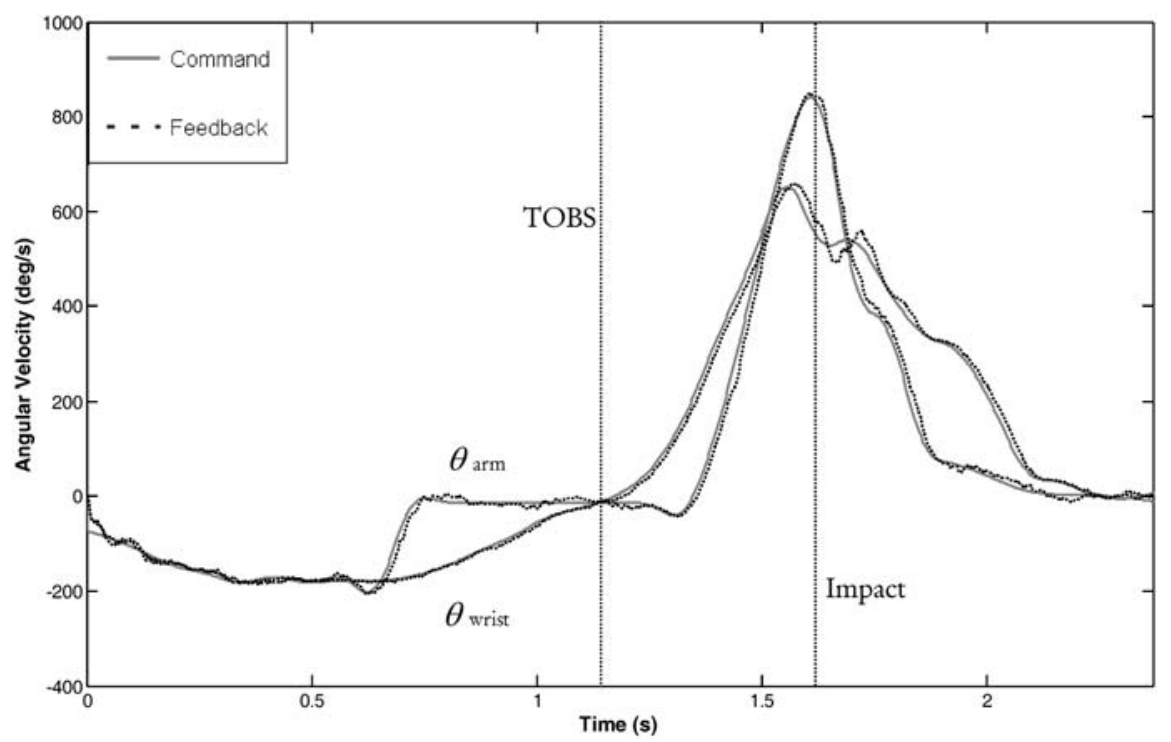

Fig. 10 Angular velocities achieved by the Robo5 (feedback data) compared with the command file for the simulation of the professional golfer's swing

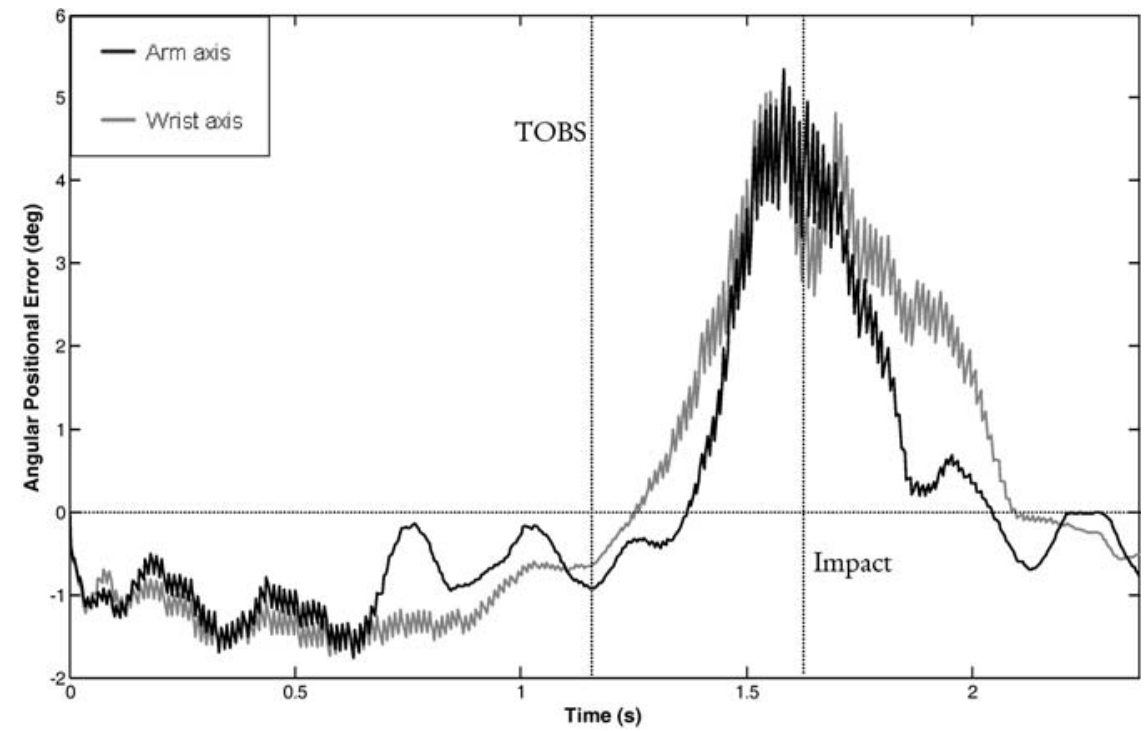

Fig. 11 Difference between command data and feedback data during the simulation of the professional golfer's swing

residual data, illustrated in Fig. 11, typically ranged from zero to negative two degrees in the backswing and became increasingly positive during the downswing, reaching a peak error value in both $\theta_{\text {arm }}$ and $\theta_{\text {wrist }}$ immediately before impact. This indicates that the Robo5 swing motions lagged behind the command joint positions throughout each simulation. The peak residual data typically ranged from five to eight degrees depending upon the command file used and the swing duration. The mean absolute deviations between the command and feedback data for both the $\theta_{\text {arm }}$ and $\theta_{\text {wrist }}$ axes were consistently less than two degrees for all of the swing simulations performed by the Robo5, and the standard deviations of these values were tiny. This indicates that a high level of performance accuracy and repeatability were achieved using the new motion control system.

Swing simulations from the derivative-fitted swing commands were smoother and less convulsive than the position-fitted swing commands but the impact speed of the clubhead and the closeness of fit between the swing command data and the discrete data were reduced. This was expected, because the peak swing velocities forecast using PD indicated decreased angular velocities when the interpolation fit was applied to higher-order derivative data. As 
shown in Fig. 8(b) for the simulation of the professional golfer's swing; the reduction in clubhead speed was less than $2 \mathrm{~m} / \mathrm{s}$ but for the Robo3 simulation the effect was much more dramatic, resulting in speed reductions of up to 50 per cent.

Derivative fitting provided a useful mechanism for smoothing the derivative profiles of swing commands; however, the command file generation technique employed by PD includes three factors that limit the quality of swing commands. The first limitation is that discrete data points must be equally spaced in time, which means that critical phases of the swing, such as the downswing, compose less than 15 per cent of the discrete data, and the motions that characterize an individual golfer's swing risk being lost. Second, the total number of discrete joint positions that may be entered into PD is limited to 40 , which prevents better representation of critical swing phases by increasing the number of discrete data points. Lastly, the curve construction method employed by PD frequently requires additional joint positions to be added after the final discrete joint position to achieve the 1000 joint positions required. The addition of joint positions after the final discrete coordinate compresses the discrete data and results in a shift of the profile shape towards the start of the swing. As a result, the technique used to generate command files could be further refined.

\section{CONCLUSION}

Three-dimensional kinematic analysis of golfers' swings has indicated that individual golfers' perform consistent swing motions, but that variability exists between the swing profiles of individual players. The swing motion of a traditional golf robot was found to provide a poor representation of golfers' swings. A commercially available golf swing robot with a modified motion control system was shown to be capable of performing variable golf swing profiles with high degrees of repeatability. A methodology for creating robot commands files from an individual golfer's kinematic swing data was demonstrated. This study has shown that more accurate mechanical simulations of individual golfers' swings may now be achieved, which has large potential benefits for manufacturers in the development of customized golf equipment. Using this approach, equipment developers will be able to test, evaluate, and refine the performance of equipment for different styles of swing.

\section{REFERENCES}

1 Cochran, A. and Stobbs, J. Search for the perfect swing, 1968 (Triumph Books, Chicago).

2 Wilson, P. Swing machine golf, 2002 (StoryTrend Publishing, Thornhill, ON).

3 Leadbetter, D. The golf swing, 1990 (Stephen Greene Press, Lexington, MA).

4 Garden Hendry, w. The dynamic anatomy of the golf swing, 1985 (Parthenon Press, Carnforth).

5 Milburn, P. D. Summation of segmental velocities in the golf swing. Med. Sci. in Sports and Exercise, 1982, 14(1), 60-64.

6 Williams, D. The dynamics of the golf swing. $J$. Mech. Appl. Maths, 1967, 20, 249-264.

7 Bunn, J. W. Scientific principles of coaching, 2nd edition, 1972, pp. 263-279 (Prentice Hall, Englewood Cliffs, NJ).

8 Putnam, C. Sequential motions of body segments in striking and throwing skills: descriptions and explanations. J. Appl. Biomech., 1993, 26, 125-135.

9 Reinsch, C. Smoothing by spline functions. $\mathrm{Nu}$ merische Mathematik, 1967, 10(3), 177-183.

10 Simon, D. Data smoothing and interpolation using eighth order algebraic splines. IEEE Trans. Signal Processing, 2004, 52(4), 1136-1144.

11 Ming, A. and Kajitani, M. A new golf swing robot to simulate human skill - accuracy improvement of swing motion by learning control. Mechatronics, 2003, 13(8-9), 809-823. 